\title{
Switched Control of Satellites for Global Stabilization and Local Performance: a Sum of Squares Approach
}

\author{
Narendra Gollu \\ Department of Mechanical and Industrial Engineering \\ Concordia University \\ Montreal, QC, H3G 2W1, Canada \\ ngolludencs. concordia.ca
}

\begin{abstract}
The main contribution of this chapter is the development of a two step switched approach for global stabilization and local performance of large angle maneuvers of satellites. The first step consists of parameterizing the attitude dynamics of the satellite by modified Rodriguez parameters (MRP) and searching for a Lyapunov-based stabilizing controller using Sum of Squares (SOS). In the second step, when the rigid body is close to its desired attitude set point, the control switches to a linear controller that can guarantee local performance and that uses less computational operations to be implemented in a microprocessor. The effectiveness of the proposed control technique is shown in simulations for a large angle maneuver corresponding to a satellite rotation about a fixed axis.
\end{abstract}

\section{INTRODUCTION}

Rigid-body attitude control is one of the most widely studied problems within nonlinear control theory, largely because of its importance in applications such as robotics, high performance aircraft, underwater vehicles and spacecraft. In spacecraft applications, satellites are often required to perform rapid and large angle maneuvers for various missions, such as for multi-target acquisition or for pointing and tracking capabilities. These rapid maneuvers are characterized by nonlinear rigid body dynamics because of the cross coupling of angular velocity terms. To account for this nonlinear coupling, many researchers have applied various nonlinear control design approaches to meet these challenges, specifically for spacecraft applications. George Meyer [1] lays the foundations for the work on spacecraft modeling by providing spacecraft dynamic models. Tsiotras [2], [3], [4] extends these results using a Lyapunov function that involves the sum of a quadratic term in the angular velocities and a logarithmic term in the kinematic parameters leading to the design of linear controllers. Crassidis et al. [5] is the first reference to consider the problem of controlling a spacecraft without full state feedback. The controller is designed by minimizing the norm-squared local errors between the predicted and desired quantities. A Lyapunovbased adaptive controller that estimates external torques has been developed by Schaub et. al. [6]. Lim [7] developed a linear parameter varying controller, in which a single quadratic Lyapunov function for each frozen linear time invariant (LTI) system was used in a parameter variation set. Raymond and Johan [8] used integrator backstepping design for satellite attitude control based on quaternions. In previous work of the authors [9], a two step integrated and systematic approach for modelling and control of large angle attitude maneuvers of a rigid body is developed.

Notice however that the cost, the processor workload and the time-constraints in spacecraft development and deployment projects curtail the opportunity for implementing nonlinear control laws during entire missions. For example in a pointing mission the satellite is always required to point toward either the Sun or the Zenith. In such a mission, nonlinear control is only needed during the acquisition mode [10]. This motivates the use of switching between a global nonlinear controller for acquisition mode and a local linear controller for pointing mode that can guarantee performance and that uses less computational operations to be implemented in a microprocessor.

While the problem of attitude control and stabilization has been a subject of much research, the problem of switching between a stabilizing nonlinear controller and a performance linear controller has not been explicitly considered for spacecraft to the best of our knowledge. In fact, designing a controller that has both a large region of attraction and a good local performance has been considered one of the most interesting research problems in nonlinear control theory [11]. It is fair to say that much of the switching work in the literature is between linear models. However, switching between a nonlinear controller and a local linear controller was considered previously to implement anti-windup controllers [12] and to swing up a pendulum [13]. In [14] a fuzzy controller was used for swinging up a pendulum and a linear state feedback controller was used for balancing the pendulum in the upper position.

In this paper, a switching controller is proposed between acquisition and pointing modes of a satellite maneuver that can achieve both global stabilization and local performance. The paper is divided into five parts and is organized as follows. Section 2 describes briefly rigid body attitude kinematics and dynamics. Section 3 formulates stabilizing controller synthesis as an SOS feasibility problem and performance controller synthesis as a linear quadratic regulator problem. Section 4 presents the switching law. Finally, section 5 presents a numerical example. 


\section{Attitude Kinematics And Dynamics}

This section presents a brief review of the kinematics and dynamics equations of motion for a three-axis stabilized spacecraft using MRP.

\section{A. Modified Rodriguez Parameters (MRP)}

The MRP are a recent addition to the family of attitude representations and are particularly well suited for describing very large attitudes [10]. The MRP are able to describe an orientation with only three parameters, instead of the four parameters required by quaternions. Let $\boldsymbol{\Phi}$ denote the principal angle and let $\hat{\mathbf{e}}=\left(e_{1}, e_{2}, e_{3}\right)$ denote the principal axis associated with Euler's theorem [10]. The Euler parameters are then defined by

$$
\begin{aligned}
& q_{1}=e_{1} \sin (\boldsymbol{\Phi} / 2) \\
& q_{2}=e_{2} \sin (\mathbf{\Phi} / 2) \\
& q_{3}=e_{3} \sin (\mathbf{\Phi} / 2) \\
& q_{4}=\cos (\mathbf{\Phi} / 2)
\end{aligned}
$$

where the first three elements, which indicate the direction of the Euler axis, are usually grouped together and written as $\mathbf{q}_{13}=\hat{\mathbf{e}} \sin (\boldsymbol{\Phi} / 2)$. The fourth element, $q_{4}$, is commonly referred to as the scalar component of the quaternion vector and indicates the principal angle. The MRP vector $\sigma$ can be expressed in terms of the four quaternion elements $\left(q_{1}, q_{2}, q_{3}, q_{4}\right)$ as

$$
\boldsymbol{\sigma}=\left[\begin{array}{l}
q_{1} /\left(1+q_{4}\right) \\
q_{2} /\left(1+q_{4}\right) \\
q_{3} /\left(1+q_{4}\right)
\end{array}\right] .
$$

It can be seen from these equations that the MRP representation has a geometric singularity at $\mathbf{\Phi}= \pm 360^{\circ}$, which corresponds to $q_{4}=-1$ in (2). Thus, any rotation less than a complete revolution can be expressed using these parameters. Note however that complete revolutions are generally not encountered in most attitude maneuvers as the spacecraft would end in the same orientation as it started. The kinematics differential equation can be written in terms of the MRPs [15] as

$$
\dot{\boldsymbol{\sigma}}=\Omega(\boldsymbol{\sigma}) \boldsymbol{\omega},
$$

where $\boldsymbol{\omega}=\left[\begin{array}{lll}\omega_{x} & \omega_{y} & \omega_{z}\end{array}\right]^{T}$ are the angular velocities of the satellite about each of the principal body axes,

$\Omega(\boldsymbol{\sigma})=\frac{1}{4}\left[\begin{array}{lll}1-\sigma^{2}+2 \sigma_{1}^{2} & 2\left(\sigma_{1} \sigma_{2}-\sigma_{3}\right) & 2\left(\sigma_{1} \sigma_{3}-\sigma_{2}\right) \\ 2\left(\sigma_{2} \sigma_{1}-\sigma_{3}\right) & 1-\sigma^{2}+2 \sigma_{2}^{2} & 2\left(\sigma_{2} \sigma_{3}-\sigma_{1}\right) \\ 2\left(\sigma_{3} \sigma_{1}-\sigma_{2}\right) & 2\left(\sigma_{3} \sigma_{2}-\sigma_{1}\right) & 1-\sigma^{2}+2 \sigma_{3}^{2}\end{array}\right]$

and $\sigma^{2}=\sigma_{1}^{2}+\sigma_{2}^{2}+\sigma_{3}^{2}$. The MRP will be used throughout the remainder of this paper for the attitude representation. The next subsection describes the attitude dynamics equations.

\section{B. Attitude Dynamics}

The attitude dynamics are given by the Newton-Euler's moment equations [16] expressed in principal axes as

$$
\begin{aligned}
I_{x} \dot{\omega}_{x}+\left(I_{z}-I_{y}\right) \omega_{y} \omega_{z} & =T_{x} \\
I_{y} \dot{\omega}_{y}+\left(I_{x}-I_{z}\right) \omega_{z} \omega_{x} & =T_{y} \\
I_{z} \dot{\omega}_{z}+\left(I_{y}-I_{x}\right) \omega_{x} \omega_{y} & =T_{z}
\end{aligned}
$$

where $\mathbf{u}=\left[\begin{array}{lll}T_{x} & T_{y} & T_{z}\end{array}\right]^{T}$ are the control torques acting on the satellite, and the principal moments of inertia $I_{x}, I_{y}$ and $I_{z}$ are the components of the inertia tensor $\mathbf{I}$ given by

$$
\mathbf{I}=\left[\begin{array}{ccc}
I_{x} & 0 & 0 \\
0 & I_{y} & 0 \\
0 & 0 & I_{z}
\end{array}\right]
$$

The state variable equations are obtained from combining equations (3) and (4) and can be written in the form

$$
\dot{\mathbf{x}}=\mathbf{f}(\mathbf{x})+\mathbf{B u},
$$

where the state vector $\mathbf{x}=\left[\omega_{x} \omega_{y} \omega_{z} \sigma_{1} \sigma_{2} \sigma_{3}\right]^{T}$ containing the angular velocities and the MRP, and the inputs torque vector $\mathbf{u}=\left[\begin{array}{lll}T_{x} & T_{y} & T_{z}\end{array}\right]^{T}$,

$$
\left[\begin{array}{c}
\dot{\omega}_{x} \\
\dot{\omega}_{y} \\
\dot{\omega}_{z} \\
\dot{\sigma}_{1} \\
\dot{\sigma}_{2} \\
\dot{\sigma}_{3}
\end{array}\right]=\left[\begin{array}{c}
f_{1} \\
f_{2} \\
f_{3} \\
f_{4} \\
f_{5} \\
f_{6}
\end{array}\right]+\left[\begin{array}{ccc}
1 / I_{x} & 0 & 0 \\
0 & 1 / I_{y} & 0 \\
0 & 0 & 1 / I_{z} \\
0 & 0 & 0 \\
0 & 0 & 0 \\
0 & 0 & 0
\end{array}\right]\left[\begin{array}{c}
T_{x} \\
T_{y} \\
T_{z}
\end{array}\right]
$$

where $f(x)$ is

$$
\begin{gathered}
f_{1}=\left[\left(I_{y}-I_{z}\right) / I_{x}\right] \omega_{y} \omega_{z}, \quad f_{2}=\left[\left(I_{z}-I_{x}\right) / I_{y}\right] \omega_{z} \omega_{x} \\
f_{3}=\left[\left(I_{x}-I_{y}\right) / I_{z}\right] \omega_{x} \omega_{y} \\
\begin{array}{r}
f_{4}=\frac{1}{4}\left[1-\sigma^{2}+2 \sigma_{1}^{2}\right] \omega_{x}+\frac{1}{4}\left[2\left(\sigma_{1} \sigma_{2}-\sigma_{3}\right)\right] \omega_{y} \\
+\frac{1}{4}\left[2\left(\sigma_{1} \sigma_{3}-\sigma_{2}\right)\right] \omega_{z} \\
f_{5}=\frac{1}{4}\left[2\left(\sigma_{2} \sigma_{1}-\sigma_{3}\right)\right] \omega_{x}+\frac{1}{4}\left[1-\sigma^{2}+2 \sigma_{2}^{2}\right] \omega_{y} \\
+\frac{1}{4}\left[2\left(\sigma_{2} \sigma_{3}-\sigma_{1}\right)\right] \omega_{z} \\
f_{6}=\frac{1}{4}\left[2\left(\sigma_{3} \sigma_{1}-\sigma_{2}\right)\right] \omega_{x}+\frac{1}{4}\left[2\left(\sigma_{3} \sigma_{2}-\sigma_{1}\right)\right] \omega_{y} \\
+\frac{1}{4}\left[1-\sigma^{2}+2 \sigma_{3}^{2}\right] \omega_{z}
\end{array}
\end{gathered}
$$

\section{Linearized Dynamics}

Linearizing the nonlinear system (6) around an equilibrium point yields a linear state space model

$$
\dot{\mathbf{x}}=A_{l} \mathbf{x}+\mathbf{B u}
$$

where

$$
A_{l}=\left[\begin{array}{cc}
O & O \\
O & 1 / 4 I
\end{array}\right], I=\left[\begin{array}{ccc}
I_{x} & 0 & 0 \\
0 & I_{y} & 0 \\
0 & 0 & I_{z}
\end{array}\right], O=\left[\begin{array}{lll}
0 & 0 & 0 \\
0 & 0 & 0 \\
0 & 0 & 0
\end{array}\right] \text {. }
$$

\section{Problem statement:}

Given attitude dynamics (6), the problem is to design an attitude controller for large angle attitude maneuvers of a satellite, a linear controller for local performance and a stabilizing switching rule that switches between the global nonlinear controller and the local linear controller. The following steps are used in solving the problem.

1) Design a nonlinear controller for the nonlinear system (6) using sum of squares. 
2) Design a linear controller for the linearized model (8) using LQR.

3) Using the control Lyapunov function obtained in point 1 , and the controller obtained in point 2 , find the largest region of attraction.

4) Propose a switching strategy between the nonlinear controller and the linear controller that switches controllers once this region of attraction is reached.

\section{StABILIZING CONTROLLER DESIGN}

The controller design approach is broken up into two steps. The first part involves the design of a controller that performs the large angle maneuver. The second part involves the design of an optimal state feedback controller for the linearized model that will stabilize the satellite around the equilibrium position and guarantees that a performance measure is achieved.

\section{A. Nonlinear Controller Design}

The nonlinear controller design will now be formulated as an optimization program. The formulation consists of the two steps described next.

1) Model Parameterization: The model parameterization consists of rewriting the equations (7) in the form

$$
\dot{\mathbf{x}}=A(\mathbf{x}) \mathbf{x}+\mathbf{B u},
$$

with

$$
A(\mathbf{x})=\left[\begin{array}{cccccc}
0 & a_{12} & 0 & 0 & 0 & 0 \\
0 & 0 & a_{23} & 0 & 0 & 0 \\
a_{31} & 0 & 0 & 0 & 0 & 0 \\
a_{41} & a_{42} & a_{43} & 0 & 0 & 0 \\
a_{51} & a_{52} & a_{53} & 0 & 0 & 0 \\
a_{61} & a_{62} & a_{63} & 0 & 0 & 0
\end{array}\right]\left[\begin{array}{c}
\omega_{x} \\
\omega_{y} \\
\omega_{z} \\
\sigma_{1} \\
\sigma_{2} \\
\sigma_{3}
\end{array}\right]
$$

where

$$
\begin{array}{llrl}
a_{12} & =\left[\left(I_{y}-I_{z}\right) / I_{x}\right] \omega_{z}, & a_{23} & =\left[\left(I_{y}-I_{z}\right) / I_{x}\right] \omega_{z} \\
a_{31} & =\left[\left(I_{x}-I_{y}\right) / I_{z}\right] \omega_{y}, & a_{41} & =\frac{1}{4}\left[1-\sigma^{2}+2 \sigma_{1}^{2}\right] \\
a_{42} & =\frac{1}{4}\left[2\left(\sigma_{1} \sigma_{2}-\sigma_{3}\right)\right], & a_{43} & =\frac{1}{4}\left[2\left(\sigma_{1} \sigma_{3}-\sigma_{2}\right)\right] \\
a_{51} & =\frac{1}{4}\left[2\left(\sigma_{1} \sigma_{3}-\sigma_{2}\right)\right], & a_{52} & =\frac{1}{4}\left[1-\sigma^{2}+2 \sigma_{2}^{2}\right] \\
a_{53} & =\frac{1}{4}\left[1-\sigma^{2}+2 \sigma_{2}^{2}\right], & a_{61} & =\frac{1}{4}\left[2\left(\sigma_{3} \sigma_{1}-\sigma_{2}\right)\right] \\
a_{62} & =\frac{1}{4}\left[2\left(\sigma_{3} \sigma_{2}-\sigma_{1}\right)\right], & a_{63} & =\frac{1}{4}\left[1-\sigma^{2}+2 \sigma_{3}^{2}\right] .
\end{array}
$$

For this model parameterization, a quadratic control Lyapunov function is proposed to perform the controller synthesis.

2) SOS Controller Design: This step involves designing a Lyapunov-based controller using SOS. To design the controller we consider a candidate control Lyapunov function for $Q>0$, of the form

$$
V(\mathbf{x})=\mathbf{x}^{T} Q \mathbf{x},
$$

where $Q=P^{-1}$. Since $Q>0$, we have $V(x)>0$. Differentiating the Lyapunov function (11) yields

$$
\dot{V}=(A(\mathbf{x}) \mathbf{x}+\mathbf{B u})^{T} Q \mathbf{x}+\mathbf{x}^{T} Q(A(\mathbf{x}) \mathbf{x}+\mathbf{B u})
$$

Assuming a control input $\mathbf{u}$ of the form

$$
\mathbf{u}(\mathbf{x})=K(\mathbf{x}) \mathbf{z}
$$

with $\mathbf{z}=P^{-1} \mathbf{x}$ and substituting into equation (12) yields

$$
\begin{aligned}
\dot{V}= & \mathbf{x}^{T}\left(A(\mathbf{x})+\mathbf{B} K(\mathbf{x}) P^{-1}\right)^{T} P^{-1} \mathbf{x} \\
& +\mathbf{x}^{T} P^{-1}\left[A(\mathbf{x})+\mathbf{B} K(\mathbf{x}) P^{-1}\right] \mathbf{x}
\end{aligned}
$$

Using $\mathbf{x}=P \mathbf{z}$, equation (14) can be rewritten as

$$
\dot{V}=z^{T}\left[P A(\mathbf{x})^{T}+A(\mathbf{x}) P+(\mathbf{B} K(\mathbf{x}))^{T}+\mathbf{B} K(\mathbf{x})\right] z
$$

Note that $\dot{V}$ is a polynomial function and the condition that must be imposed to this polynomial for asymptotic stability is $\dot{V}<0$. However, it is well known that verifying that a given polynomial is non-negative is in general an NP hard problem [17]. Therefore, a relaxation of the non-negativity condition proposed by Parrilo [17] is to limit the use of polynomials to a special form that is known to be positive semi-definite: sums of squares (SOS). A brief summary of sum of squares technique is discussed below. For $\mathbf{x} \in \Re^{n}$, a multivariate polynomial $p(\mathbf{x})$ is a sum of squares if there exist some polynomials $f_{i}(\mathbf{x}), i=1, \ldots, M$, such that [18]

$$
p(\mathbf{x})=\sum_{i=1}^{M} f_{i}^{2}(\mathbf{x}) .
$$

A polynomial $p(\mathbf{x})$ of degree $2 d$ is a sum of squares if and only if there exists a positive semidefinite matrix $Q$ and a vector of monomials $Z(\mathbf{x})$ containing monomials in $\mathbf{x}$ of degree less than $d$ such that [18]

$$
p(\mathbf{x})=Z(\mathbf{x})^{T} Q Z(\mathbf{x}) .
$$

It should be noted that $p(\mathbf{x})$ being a sum of squares implies that $p(\mathbf{x}) \geq 0$, but the converse is generally not true. Using this relaxation the condition $\dot{V}(x)<0$ on the Lyapunov function $V(x)$ can be replaced by SOS constraints. A simplified version of a Theorem from [19] is stated that will be useful to prove the condition (15) is asymptotically stable.

Theorem 3.1: [19] For the system (10), suppose there exists $P>0$, a polynomial matrix $K(x)$, and a sum of squares $\epsilon(x)$, such that the following expression

$-v^{T}\left(P A^{T}(x)+A(x) P+K^{T}(x) \mathbf{B}^{T}(x)+\mathbf{B}(x) K(x)+\epsilon(x) I\right) v$,

is SOS, where $v \in \Re^{n}$. Then the state feedback stabilization problem is solvable, and a controller that stabilizes the system is given by

$$
\mathbf{u}(\mathbf{x})=K(\mathbf{x}) P^{-1} \mathbf{x} .
$$

Furthermore, if equation (18) holds with $\epsilon(x)>0$ for $x \neq 0$, then the zero equilibrium is globally asymptotically stable.

Proof: It follows from the proof of [19] with $P(\tilde{x})=$ $P, Z(x)=x$ and $M=I$.

Based on theorem 3.1, and the relaxations using sum of squares technique the following control optimization problem is defined. 
Definition 3.1: The attitude control design optimization problem is:

$$
\begin{aligned}
\text { find } & P, K(\mathbf{x}) \\
\text { s.t. } & \left(P-\epsilon_{1} I\right)>0, \epsilon_{1}>0, \epsilon_{2}(x) \text { is } \mathrm{SOS} \\
- & z^{T}\left[P A^{T}+A P+(\mathbf{B} K)^{T}+\mathbf{B} K\right] z-\epsilon_{2}\|\mathbf{x}\|^{2} \text { is SOS }
\end{aligned}
$$

where $I$ is an identity matrix. The next section will present the linear controller design.

\section{B. Linear Controller Design}

The state feedback controller responsible for maintaining the satellite at a given position is based on a Linear Quadratic Regulator (LQR) [20] design using the linearized system (8). In a LQR design, the control input is found by minimizing a quadratic cost function of the form

$$
J=\int_{0}^{\infty} x(t)^{T} Q x(t)+u(t)^{T} R u(t) d t
$$

where $Q$ and $R$ are weighting parameters that penalize the energy on the states and control inputs, respectively. The solution to this problem is the control law

$$
\mathbf{u}_{l}=\mathbf{k}_{l} \mathbf{x}
$$

that stabilizes the system around the linearized point and guarantees the required performance is achieved. Since this control law control law is based on the linearized system, the state feedback optimal controller is only effective when the system is close to the linearizing point, i.e, when the satellite is in the pointing mode.

\section{SWitching LAw Between Global And Local CONTROLler}

The objective of this section is to formulate a switching strategy to switch from the globally stabilizing controller in acquisition mode to the local performance controller in pointing mode. We propose here that the switching occurs when the satellite approaches the maximum attractive region for the linear controller that can be estimated using the quadratic Lyapunov function (11). Given this Lyapunov function and the linear controller (21), the objective is therefore to find the largest invariant set

$$
\Omega_{\alpha}=\left\{\mathbf{x} \in \Re^{n} \mid V(\mathbf{x})<\alpha\right\}
$$

for the nonlinear closed loop system which is obtained by substituting the linear controller (21) into equation (10)

$$
\dot{\mathbf{x}}=A(\mathbf{x}) \mathbf{x}+\mathbf{B} \mathbf{k}_{l} \mathbf{x}
$$

Based on Khalil ([21], pg. 122), if there is a Lyapunov function that satisfies the conditions of asymptotic stability over a bounded domain $D$ and if $\Omega_{\alpha}$ is contained in $D$, then every trajectory starting in $\Omega_{\alpha}$ remains in $\Omega_{\alpha}$ and approaches the origin as $t \rightarrow \infty$. Using the dynamics (23), the derivative of the candidate Lyapunov function (11) along the trajectories of the system is

$$
\dot{V}_{c}=\mathbf{x}^{T}\left[A^{T}(\mathbf{x}) Q+Q A(\mathbf{x})+\left(\mathbf{B} k_{l}\right)^{T}+\mathbf{B} k_{l}\right] \mathbf{x}
$$

A sufficient condition for estimating the largest region of attraction can now be formulated as the following optimization problem.

Definition 4.1: Given Q, $k_{l}$

$$
\begin{aligned}
\max & \alpha \\
\text { s.t. } & \epsilon_{3}>0, s(\mathbf{x}) \text { is } S O S \\
& -s(x)(V(x)-\alpha) \text { is } S O S \\
& -\dot{V}_{c}-\epsilon_{3}\|\mathbf{x}\|^{2}+s(\mathbf{x})(V(\mathbf{x})-\alpha) \text { is } S O S
\end{aligned}
$$

The above optimization problem 4.1 is biconvex. With the recent introduction of YALMIP [22] and PENBMI [23], which allows to solve locally biconvex optimization problem, we can solve the above optimization problem resulting in a region

$$
\Omega_{\alpha^{*}}=\left\{\mathbf{x} \in \Re^{n} \mid V(\mathbf{x})<\alpha^{*}\right\}
$$

where $\Omega_{\alpha}^{*}$ is the largest region of attraction that can be found numerically.

Given a nonlinear controller (19) for the system (10), a linear controller (21) designed for the linearized system (8) , and the largest region of attraction (25) for the nonlinear closed loop system (23), the switching between the controllers happens when the states enter the largest region of attraction. The linear controller is used when the states are inside the region of attraction and the nonlinear controller is used when the states are outside the region of attraction.

$$
U= \begin{cases}k_{l} \mathbf{x}, & \text { if } \mathbf{x} \in \Omega_{\alpha^{*}} \\ K(\mathbf{x}) Q \mathbf{x}, & \text { otherwise. }\end{cases}
$$

Theorem 4.1: For system (10), let there exist a global control Lyapunov function of the form (11), a nonlinear controller (19), and a linear controller (21) for the linearized system (8), then the system (10) is asymptotically stable when using the switching law (26).

\section{Proof}

The proof of this theorem is divided into three parts.

1) If the states start outside the region of attraction, the states will reach this region for a finite time of $T$. To prove that the trajectories of the closed loop system (24) converge to a region $\Omega_{\alpha^{*}}$, we use the comparison lemma [21]. From the conditions in Definition 3.1, we have

$$
-\dot{V}(\mathbf{x})-\epsilon_{2}\|\mathbf{x}\|^{2} \text { is } S O S
$$

which implies

$$
\dot{V}(\mathbf{x}) \leq-\epsilon_{2}\|\mathbf{x}\|^{2}
$$

Given that $V(\mathbf{x})=\mathbf{x}^{T} Q \mathbf{x}<\lambda_{\max }(Q)\|\mathbf{x}\|^{2}$, we have

$$
\dot{V}(\mathbf{x})<-\beta V(\mathbf{x})
$$

where $\beta=\frac{\epsilon_{2}}{\lambda_{\max }(Q)}$ is the decay rate and $\lambda_{\max }(Q)$ is the maximum eigen value of $Q$. Using the comparison lemma, we have $V(t)<V(0) e^{-\beta t}$. Let $T$ be the first time, possibly infinite, at which trajectories reach $\Omega_{\alpha^{*}}$. Then, from

$$
V(t)<V(0) e^{-\beta t}
$$




$$
T<-\frac{1}{\beta} \ln \frac{\alpha^{*}}{V(0)}
$$

Thus, from equation (27) we can conclude that the system trajectories converge to the region no later than time $T$.

2) If the states start inside the region of attraction, by the definition of the region of attraction, they remain in the region and asymptotically converge to the origin since $(V(\mathbf{x}))<-\epsilon_{3}\|\mathbf{x}\|^{2}$.

3) If the states start at the boundary of the region of attraction, then the states will enter into the region of attraction, because the closed loop vector fields corresponding to either the nonlinear controller or the linear controller in the loop point towards the region because of, respectively, the inequalities,

$$
\begin{aligned}
& \frac{\partial V}{\partial x} \dot{x}=\dot{V}(\mathbf{x})<-\epsilon_{2}\|\mathbf{x}\|^{2}<0 \\
& \frac{\partial V}{\partial x} \dot{x}=\dot{V}_{c}(\mathbf{x})<-\epsilon_{3}\|\mathbf{x}\|^{2}<0
\end{aligned}
$$

There will be no sliding modes or chattering because the vector fields point inwards and any convex combination of two fields has to point inwards, i.e, for $0 \leq l_{1} \leq 1$

$$
\dot{V}_{\text {hull }}<-l_{1} \epsilon_{2}\|\mathbf{x}\|^{2}-\left(1-l_{1}\right) \epsilon_{3}\|\mathbf{x}\|^{2}<0
$$

\section{NuMERICAL EXAMPLE}

The preceding theoretical results are now applied to a numerical example. The objective is to bring a rigid spacecraft with an initial nonzero attitude to rest at a zero attitude vector. We consider a rigid spacecraft with inertia parameters $I=\operatorname{diag}(140,100,80)\left(\mathrm{kg} \cdot \mathrm{m}^{2}\right)$. The initial angular velocities are zero and the initial Euler angles of the satellite are $\Theta_{0}=\left(75^{\circ},-175^{\circ}, 70^{\circ}\right)$. The desired Euler angles of the satellite are $\Theta_{d}=\left(0^{\circ} ; 0^{\circ} ; 0^{\circ}\right)$. Using MRP, $\Theta_{0}$ corresponds to $\boldsymbol{\sigma}(0)=(0.701,-0.9331,0.7624)^{T}$ and $\Theta_{d}$ to $\boldsymbol{\sigma}(d)=$ $(0,0,0)^{T}$. Substituting the inertial parameters in equation (10), $A(x)$ and $B$ become

$$
\begin{gathered}
A(\mathbf{x})=\left[\begin{array}{cccccc}
0 & 0.14 \omega_{z} & 0 & 0 & 0 & 0 \\
0 & 0 & -0.6 \omega_{x} & 0 & 0 & 0 \\
-0.5 \omega_{y} & 0 & 0 & 0 & 0 & 0 \\
a_{41} & a_{42} & a_{43} & 0 & 0 & 0 \\
a_{51} & a_{52} & a_{53} & 0 & 0 & 0 \\
a_{61} & a_{62} & a_{63} & 0 & 0 & 0
\end{array}\right] \\
B=\left[\begin{array}{cccc}
0.0070 & 0 & 0 & \\
0 & -0.0100 & 0 \\
0 & 0 & -0.0125 \\
0 & 0 & 0 \\
0 & 0 & 0 & 0
\end{array}\right]
\end{gathered}
$$

where $a_{41}, a_{42}, a_{43}, a_{51}, a_{52}, a_{53}, a_{61}, a_{62}, a_{63}$ are defined in equation (10). Given the matrices $A(\mathbf{x}), B$ and the values

$$
\begin{aligned}
& \epsilon_{1}=1, \\
& \epsilon_{2}=1,
\end{aligned}
$$

the software package SOStools [18] is used to solve the feasibility problem in Definition 3.1. The following controller and the symmetric matrix $P$ are obtained.

$$
P=\left[\begin{array}{cccccc}
19.7 & 0.0 & 0 & -39.1 & 0 & 0 \\
0 & 34.9 & 0 & 0 & -70.2 & 0 \\
0 & 0 & 20.4 & 0 & 0 & -28.4 \\
-39.1 & 0 & 0 & 127.8 & 0 & 0 \\
0 & -70.2 & 0 & 0 & 187.1 & 0 \\
0 & 0 & -28.4 & 0 & 0 & 91.3
\end{array}\right]
$$

$$
\begin{aligned}
T_{x}= & -34.1 x_{1}^{3}-13.5 x_{1}^{2} x_{4}-18.2 x_{1} x_{2}^{2}-6.8 x_{1} x_{2} x_{5} \\
- & 10.7 x_{1} x_{3}^{2}-4.1 x_{1} x_{3} x_{6}-6.3 x_{1} x_{4}^{2}-4.1 x_{1} x_{5}^{2} \\
- & 7.9 x_{1} x_{6}^{2}-41.1 x_{1}-4 x_{2}^{2} x 4-1.5 x_{2} x_{3}-1.3 x_{2} x_{4} x_{5} \\
- & 6.3 x_{2} x_{6}-2.1 x_{3}^{2} x_{4}-0.1 x_{3} x_{4} x_{6}+0.6 x_{3} x_{5}-1.6 x_{4}^{3} \\
- & 1.1 x_{4} x_{5}^{2}-2.1 x_{4} x_{6}^{2}-1.9 x_{5} x_{6} \\
T_{y}= & -29.7 x_{1}^{2} x_{2}-7.3 x_{1}^{2} x_{5}-9.6 x_{1} x_{2} x_{4}-1.4 x_{1} x_{3} \\
- & 2.1 x_{1} x_{4} x_{5}-9.9 x_{1} x_{6}-32.3 x_{2}^{3}-15.4 x_{2}^{2} x_{5} \\
- & 15.3 x_{2} x_{3}^{2}-5.8 x_{2} x_{3} x_{6}-7.4 x_{2} x_{4}^{2}-5.5 x_{2} x_{5}^{2} \\
- & 9.0 x_{2} x_{6}^{2}-43.4 x_{2}-3.9 x_{3}^{2} x_{5}+3.5 x_{3} x_{4}-1 x_{3} x_{5} x_{6} \\
- & 2.6 x_{4}^{2} x_{5}-1.6 x_{4} x_{6}-1.5 x_{5}^{3}-3.1 x_{5} x_{6}^{2}-16.4 x_{5} \\
T_{z}= & 22.4 x_{1}^{2} x_{3}-4.2 x_{1}^{2} x_{6}+0.8 x_{1} x_{2}-7.1 x_{1} x_{3} x_{4} \\
& -0.1 x_{1} x_{4} x_{6}+2.4 x_{1} x_{5}-21 x_{2}^{2} x_{3}-4.4 x_{2}^{2} x_{6} \\
& -7.8 x_{2} x_{3} x_{5}+6.4 x_{2} x_{4}-1 x_{2} x_{5} x_{6}-21.2 x_{3}^{3} \\
& -9.2 x_{3}^{2} x_{6}-5.8 x_{3} x_{4}^{2}-3.9 x_{3} x_{5}^{2}-7 x_{3} x_{6}^{2} \\
& -23 x_{3}+3 x_{4} x_{5}-0.9 x_{5}^{2} x_{6}-2 x_{6}^{3}-7.6 x_{6}
\end{aligned}
$$

Now, we simulate the results for linearized system 8 where $A_{l}$ and $B$ are

$$
A_{l}=\left[\begin{array}{cc}
O & O \\
O & 1 / 4 I
\end{array}\right], I=\left[\begin{array}{ccc}
140 & 0 & 0 \\
0 & 100 & 0 \\
0 & 0 & 80
\end{array}\right]
$$

and $O$ is defined in equation (8) For

$$
\begin{gathered}
Q=\left[\begin{array}{cccccc}
10 & 0 & 0 & 0 & 0 & 0 \\
0 & 10 & 0 & 0 & 0 & 0 \\
0 & 0 & 10 & 0 & 0 & 0 \\
0 & 0 & 0 & 10 & 0 & 0 \\
0 & 0 & 0 & 0 & 10 & 0 \\
0 & 0 & 0 & 0 & 0 & 10
\end{array}\right] \\
R=\left[\begin{array}{ccc}
10 & 0 & 0 \\
0 & 10 & 0 \\
0 & 0 & 10
\end{array}\right]
\end{gathered}
$$

the linear controller gain $k_{l}$ is given as

$$
k_{l}=\left[\begin{array}{cccccc}
10.5 & 0.0 & -0.0 & 0.7 & -0.05 & 0.7 \\
0.0 & 8.9 & 0.0 & -0.6 & 0.5 & 0.7 \\
-0.0 & 0.0 & 8 & -0.4 & -0.9 & 0.3
\end{array}\right]
$$

Given the nonlinear controller gains and linear controller gains, we plot the the comparison of linear controller and nonlinear controller for nonlinear system in figure 1 . From figure 1 , it is observed that the linear controller does not perform good when compared to the nonlinear controller. However, the linear controller for a linearized 
system performs better for the same conditions. In figure 2, the time response for attitude parameters is plotted using the switching law (26), where $\alpha^{*}=0.9$. The value of $\alpha^{*}$ is obtained by solving the optimization problem 4.1.It is observed that the switching happens after 10 seconds and the attitude parameters converge to the desired points.
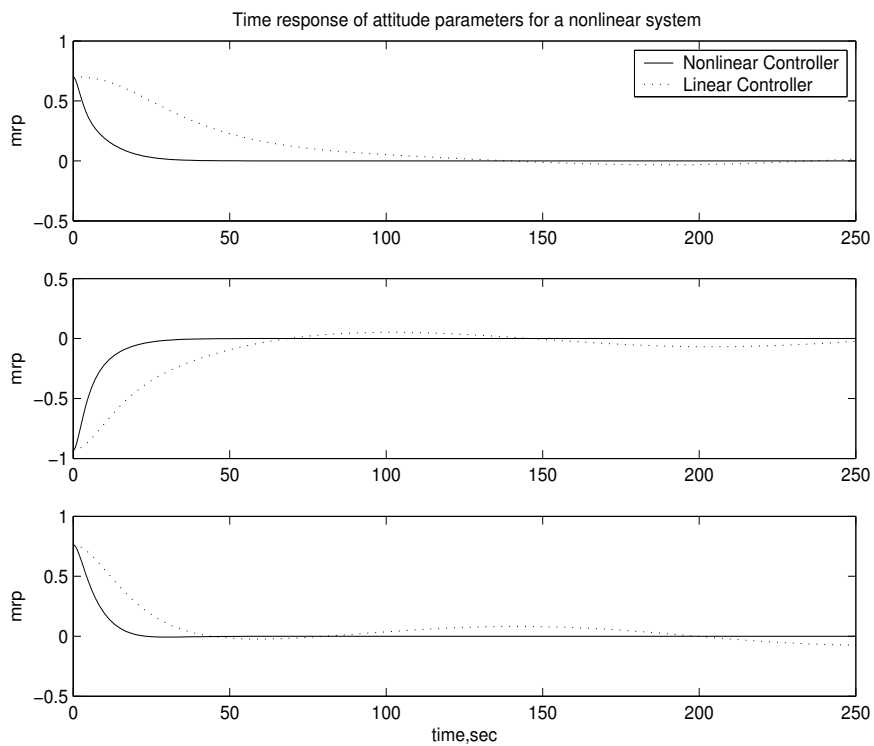

Fig. 1. Comparison of attitude $\sigma$ using nonlinear and linear controller
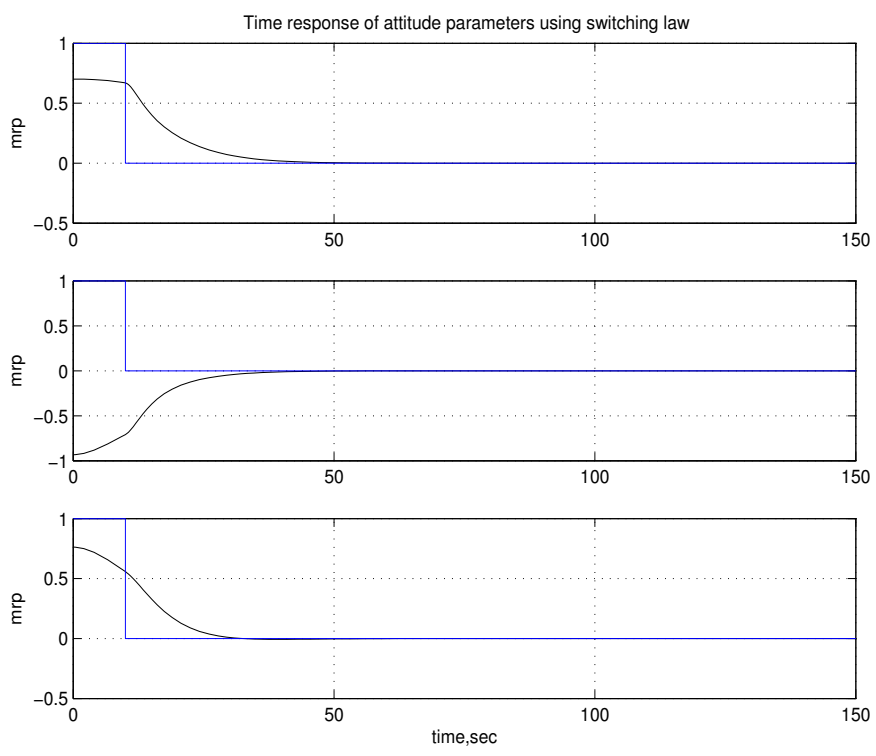

Fig. 2. Time response of attitude $\boldsymbol{\sigma}$ using switching law

\section{CONCLUSiOnS}

The main contribution of this paper was the development of a two step switched approach for global stabilization and local performance of large angle maneuvers of satellites. The first step consists of parameterizing the attitude dynamics of the satellite by modified Rodriguez parameters (MRP) and searching for a Lyapunov-based stabilizing controller using Sum of Squares (SOS). In the second step, when the rigid body is close to its desired attitude set point, the control switches to a linear controller that can guarantee local performance and that uses less computational operations to be implemented in a microprocessor. This enables possible implementation in future satellite missions without processor overload.

\section{ACKNOWLEDGMENTS}

The authors would like to acknowledge Dr. Kim and Dr. Ng of the Canadian Space Agency for many interesting and helpful discussions.

\section{REFERENCES}

[1] G. Meyer, "Design and global analysis of spacecraft attitude control," tech. rep., NASA TR R-361, 1971.

[2] P. Tsiotras, "New control laws for the attitude stabilization of rigid bodies," 13th IFAC Symposium on Automatic Control in Aerospace, pp. 316-321, 1994.

[3] P. Tsiotras, "Stabilization and optimality results for the attitude control problem," AIAA Journal of Guidance, Control and Dynamics, vol. 19, no. 4, pp. 772-779, 1996.

[4] P. Tsiotras, "Further passivity results for the attitude control problem," IEEE Transactions on Automatic Control, vol. 43, no. 11, pp. 15971600, 1998.

[5] J. L. Crassidis, F. L. Markley, T. C. Anthony, and S. F. Andrews, "Nonlinear predictive control of spacecraft," 35th Aerospace Sciences Meeting and Exhibit, 1997.

[6] H. Schaub, J. L. Junkins, and R. D. Robinett, "Adaptive external torque estimation by means of tracking a lyapunov function," AAS/AIAA Space Flight Mechanics Meeting, 1996.

[7] S. Lim and K. Chan, "Quaternion controller design using switching linear parameter varying framework," AIAA Journal of Guidance, Control and Dynamics, vol. 26, no. 3, 2003.

[8] K. Raymond and N. P. Johan, "Satellite attitude control by quaternionbased backstepping," IEEE Proceedings American Control Conference, 2005.

[9] N. Gollu and L. Rodrigues, "Control of large angle attitude maneuvers for rigid bodies using sum of squares," IEEE Proceedings American Control Conference, 2006.

[10] H. Schaub and J. L. Junkins, Analytical Mechanics of Aerospace Systems. AIAA, 2003.

[11] R. M. Murray, Top ten research problems in nonlinear control. 1996.

[12] C. Barbu, S. Galeani, A. R. Teel, and L. Zaccarian, "Non-linear antiwindup for manual flight control," International Journal of Control, vol. 78, no. 14, pp. 1111-1129, 2005.

[13] K. J. Astrom and K. Furata, "Swinging up a pendulum by energy control," Proceedings of the 13th IFAC World Congress, 1996.

[14] N. Muskinja and B. Tovornik, "Adaptive state controller for inverted pendulum," 9th Mediterranean Conference on Control and Automation, 2001.

[15] M. D. Shuster, "A survey of attitude representations," The Journal of the Astronautical Sciences, vol. 41, no. 4, pp. 439-517, 1993.

[16] M. J. Sidi, Spacecraft Dynamics and Control. New York: Cambridge University Press, 1997.

[17] P. A. Parrilo, Structured Semidefinite Programs and Semialgebraic Geometry Methods in Robustness and Optimization. $\mathrm{PhD}$ thesis, Massachusetts Institute of Technology, Boston, 2000.

[18] S. Prajna, A. Papachristodoulou, P. Seiler, and P. A. Parrilo, "Sostools: Sum of squares optimization toolbox for matlab," tech. rep., California Institude of Technology, Pasadena, California, 2004.

[19] S. Prajna, A. Papachristodoulou, and F. Wu, "Nonlinear control synthesis by sum of squares optimization: A lyapunov-based approach," Proceedings of the ASCC, 2004.

[20] T. Kailath, Linear Systems. Prentice Hall, 1989.

[21] H. Khalil, Nonlinear Systems. Prentice Hall, 1996.

[22] J. Löfberg, "YALMIP : A toolbox for modeling and optimization in MATLAB," in Proceedings of the CACSD Conference, (Taipei, Taiwan), 2004

[23] M. Kocvara, F. Leibfritz, M. Stingl, and D. Henrion, "A nonlinear SDP algorithm for static output feedback problems in $C O M P l_{e} i b$," tech. rep., University of Trier, Germany, 2004. 